\title{
Contiguous urban rivers should not be necessarily submitted to the same management plan: the case of Tietê and Pinheiros Rivers (São Paulo-Brazil)
}

\author{
DAVI G.F. CUNHA ${ }^{1}$, DORON GRULL ${ }^{2}$, MURILO DAMATO ${ }^{2}$, JOSÉ R.C. BLUM ${ }^{2}$, \\ SERGIO EIGER ${ }^{3}$, JOSÉ E.I. LUTTI ${ }^{4}$ and PEDRO C.S. MANCUSO ${ }^{3}$ \\ ${ }^{1}$ Departamento de Hidráulica e Saneamento, Escola de Engenharia de São Carlos, \\ Universidade de São Paulo (SHS-EESC-USP), Av. Trabalhador São-Carlense, 400, \\ 13566-590 São Carlos, SP, Brasil \\ ${ }^{2}$ Centro de Apoio à Faculdade de Saúde Pública, Universidade de São Paulo (CEAP-FSP-USP), \\ Av. Dr. Arnaldo, 715, 01246-904 São Paulo, SP, Brasil \\ ${ }^{3}$ Departamento de Saúde Ambiental, Faculdade de Saúde Pública, \\ Universidade de São Paulo (HSA-FSP-USP), Av. Dr. Arnaldo, 715, 01246-904 São Paulo, SP, Brasil \\ ${ }^{4}$ Ministério Público do Estado de São Paulo (MP-SP), Rua Riachuelo, 115, 01007-904 São Paulo, SP, Brasil \\ Manuscript received on June 8, 2009; accepted for publication on March 1, 2011
}

\begin{abstract}
The management of urban water resources plays an important role for developing countries. The Tietê and Pinheiros Rivers (São Paulo, Brazil) are affected by domestic and industrial effluents and by the diffuse pollution. This research aimed to quantify 134 variables in the water of Tietê and Pinheiros Rivers (approximately 7,200 and 6,600 analyses, respectively) from August 2007 to December 2008. The idea was to verify if the fact that both rivers are located in the same basin is enough to consider the application of a single management plan for both. Data showed that the rivers presented significant anthropogenic interference. The results suggested that such rivers must be subjected to individual management plans since there were exclusive occurrences (variables that were only detected in one of the rivers). Moreover, there was a statistically significant difference between rainy and dry periods for eleven variables ( $\mathrm{p}^{*}<0.05$, ANOVA), reinforcing the special importance of the temporal component within the monitoring program. It is expected that this study subsidize environmental recovery programs in the Tietê River, to which is recommendable to focus on prosecution of illegal wastewater releases, and in the Pinheiros River, to which special attention is suggested to the pollution derived from the pesticides load to the water body.
\end{abstract}

Key words: environmental monitoring, metropolitan region of São Paulo (MRSP), urban rivers, water pollution, water quality, watershed management.

\section{INTRODUCTION}

Urban rivers are usually submitted to various anthropogenic impacts not only on their quantitative aspects, but also on their qualitative characteristics. These effects include decreasing of the water quality, threatening of the aquatic biota, changing of the pristine conditions of flow and of other hydraulic conditions. Many recent

Correspondence to: Davi Gasparini Fernandes Cunha E-mail: davig@sc.usp.br researches have been linking land-use and vegetation presence or absence with the nutrient levels in the water bodies. Industrial, domestic effluents and runoff might contribute to the increase of heavy metals concentrations in the water, as well as organic and inorganic compounds. Besides, factors like geomorphology and climate also play an important role on the water quality (Turner and Rabalais 2003, Blanchoud et al. 2007, Galbraith and Burns 2007, Bedore et al. 2008, Göbel et al. 2007, Miserendino et al. 2008). 
Compared to non-urban aquatic systems, urban rivers tend to be more influenced by runoff from stormflows events through diffuse pollution. These stormflows are frequently able to increase total suspended solids, cadmium, copper, lead and zinc concentrations, as well as sodium and sulfate (from road deicers), and to decrease dissolved oxygen. Moreover, macroinvertebrate communities in urban rivers have few sensitive species and are dominated by tolerant species (Fuchs et al. 1997, Tong and Chen 2002, Gray 2004). One of the greatest environmental problems in developing countries is to control the diffuse pollution originated from urban and rural runoff (Tucci 2004). However, in these countries, the relative importance of diffuse pollution is smaller than the importance of pollution from point sources. Therefore, Brazilian urban rivers are subordinated not only to runoff from stormflows, but also, and mainly, to domestic and industrial discharges, which contribute to the water quality decrease. Summarily, wastewater and stormwater management is progressively becoming a complex task for the megacities around the world (Varis et al. 2006).

The Tietê and Pinheiros Rivers are two aquatic systems located in São Paulo State (Southeast Brazil). According to Abraham et al. (2007), it is assumed that untreated domestic wastewater from 10 million inhabitants is daily discharged into the Tietê River. These authors observed high concentrations of pathogenic microorganisms (E. coli, Shiguella flexneri and S. boydii) in the Tietê River (in the city of São Paulo), which could show that the poor water quality of this aquatic system might even cause negative effects on public health. Therefore, an accented pollution process has been occurring in the Tietê River since 1950 as a consequence of domestic and industrial effluents release. The Tietê River flows through São Paulo State and receives the water of the Pinheiros River in São Paulo City. The Pinheiros River's flow direction had been reversed into Billings Reservoir until 1992, in order to increase the electricity generation. After 1992, however, with the increase of the pollution process, this procedure was prohibited, except in cases of flood control in São Paulo (Braga 2000, Silva et al. 2002). In this last case, Pedreira Dam and Pumping Station convey the water from the Pinheiros River to the reservoir in order to prevent flooding in the urban area.
The main motivation for this research was to find out if two contiguous urban rivers tend to be similar or different when it comes to water quality. Thus, a consequence of this study was the analysis of the relative importance of the spatial scale (in terms of basin or sub-basin) in the handling of water resources. The Tietê and Pinheiros Rivers may be included in the same basin or, contrarily, in different sub-basins, depending on the spatial scale. The present study was performed to answer the following question: Is it possible to delineate the same water quality management plan for both rivers or is it imperative to establish different plans for each aquatic system, disregarding their spatial proximity and taking into account their peculiarities and exclusive occurrences of some water variables?

To reach an accurate answer, this study aimed to determine the specific characteristics of the water quality of these two urban rivers in São Paulo State (Brazil) through an intense monitoring program that was conducted for about seventeen months. As a specific objective, this research aimed to compare the results of the Tietê and Pinheiros Rivers, considering the contributions of the drainage sub-basins for each aquatic system. The idea was to investigate the influence and the relative importance of the land use patterns of each urban sub-basin in the presence (and, consequently, in the concentrations) or in the absence of some water quality variables in samples of each river.

\section{MATERIALS AND METHODS}

Two sampling stations were considered (Fig. 1), one of them in the Tietê River (TIE), immediately upstream to its confluence with the Pinheiros River, and other in the Pinheiros River (PIN), about $15 \mathrm{~km}$ upstream of its mouth.

Sampling campaigns were performed from August 2007 to December 2008, through the quantification of 134 biological, chemical and physical variables in the water, following APHA (2005) methods. The laboratories in charge of all the analyses were Laboratório Ambiental and Ecolabor (both certified by ABNT the Brazilian Authority on Technical Norms). The frequency of analysis varied from substance to substance (e.g. weekly, biweekly, monthly, bimonthly), but the total number of data was about 7,200 for the Tietê 

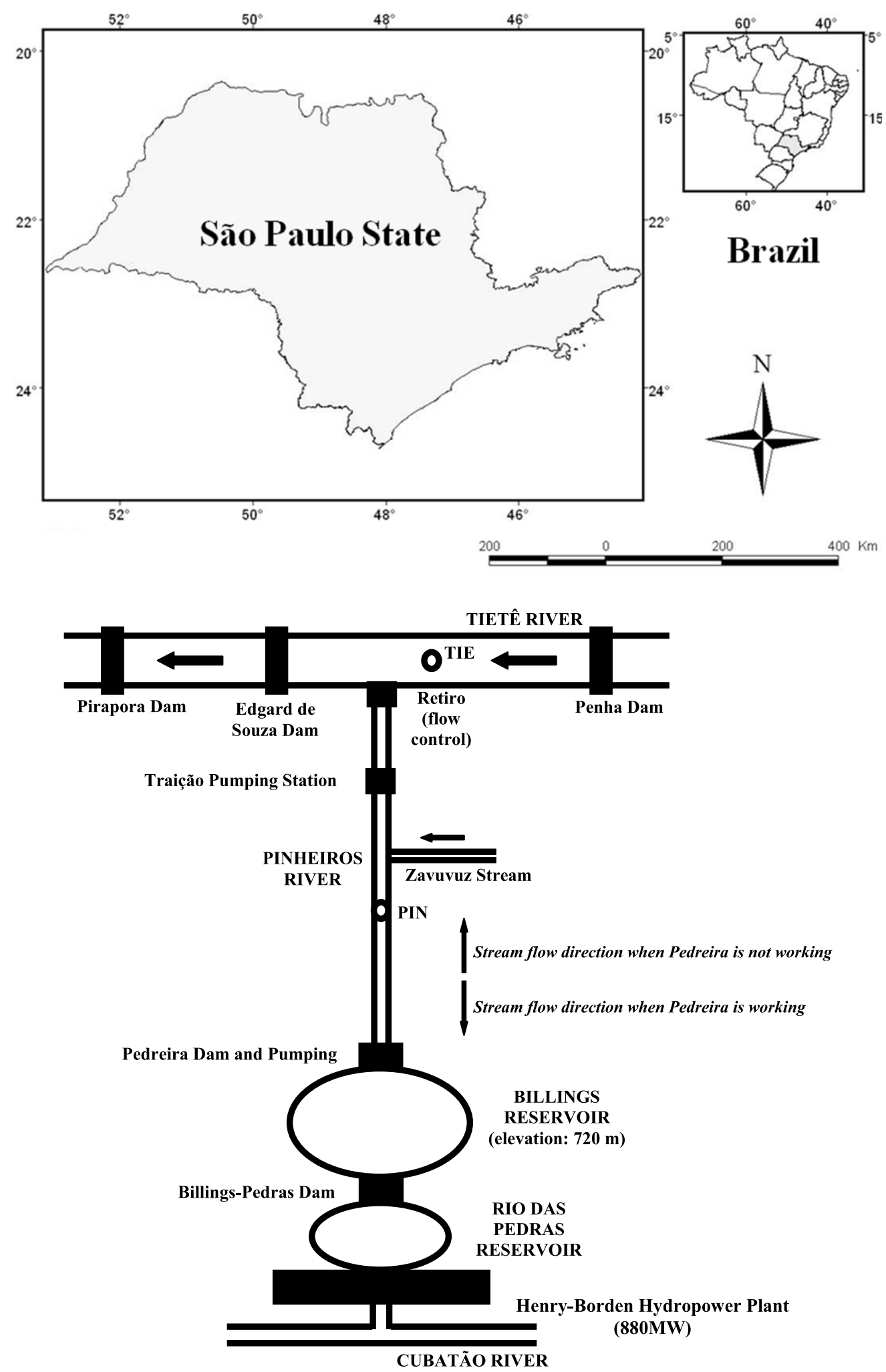

Fig. 1 - Scheme of sampling stations in the Tietê (TIE) and Pinheiros (PIN) Rivers, which are located in São Paulo State (Brazil). 
River and 6,600 for the Pinheiros River. For this reason, we present not only the mean, minimum and maximum values or concentrations of each variable, but also the coefficient of variation (C.V.) for each case in order to highlight the temporal component of the monitoring program. The C.V. was calculated by dividing the standard deviation by the mean of a given variable and multiplying the result by $100 \%$.

For practical purposes, all the variables were allocated in three possible cases according to their respective concentrations and values:

i) Case A ("exclusivity") - the variable was detected either in the Tietê River or in the Pinheiros River;

ii) Case B ("presence") - the variable was detected in both rivers:

- Case B-TIE - the maximum value was higher in the Tietê River;

- Case B-PIN - the maximum value was higher in the Pinheiros River;

iii) Case C ("absence") - the variable was detected in none of the rivers for all samples, considering the detection limit for each variable.

This division significantly helped the identification of the exclusive occurrences in each river (Case A) and the river whose sample presented the most critical value when the variable was detected in both (Case B). Also, it was useful to segregate those variables that were not observed in the aquatic systems for any sample (Case C). Variables from Cases A and B were submitted to statistical analyses in order to verify the significance of their temporal variation. Therefore, the hydrologic year 2007-2008 was divided into wet period (from October 2007 to March 2008) and dry period (from April to September 2008). The statistical procedures were conducted through the Analysis of Variance (ANOVA) for all detected water variables to assess the differences between the rainy and dry seasons, under the probability of $95 \%\left(p^{*}<0.05\right)$. The software Systat $10^{\circledR}$ was used for this purpose.

\section{RESULTS AND DISCUSSION}

The monitoring program results (Table I) are shown for those variables that presented concentrations or val- ues higher than the respective detection limit. All this data are available in the website of the Brazilian State Attorney (http://www.mp.sp.gov.br). The substances are presented in alphabetical order, with their mean, minimum, maximum concentrations and, additionally, the coefficient of variation and the total number of samples for each case. When it comes to the organic compounds, it is important to observe the high concentrations of 1.1-Dichloroethene (maximum of $13.0 \mu \mathrm{g} . \mathrm{L}^{-1}$ in the Tietê River and 17.0 $\mu \mathrm{g} . \mathrm{L}^{-1}$ in the Pinheiros River), Chloroform (maximum of $13.0 \mu \mathrm{g} . \mathrm{L}^{-1}$ in the Tietê River and $43.0 \mu \mathrm{g} . \mathrm{L}^{-1}$ in the Pinheiros River), Methylene Chloride (maximum of $75.0 \mu \mathrm{g} . \mathrm{L}^{-1}$ in the Tietê River and $15.0 \mu \mathrm{g} . \mathrm{L}^{-1}$ in the Pinheiros River) and Toluene (reaching $106.0 \mu \mathrm{g} . \mathrm{L}^{-1}$ in the Tietê River and $215.0 \mu \mathrm{g} . \mathrm{L}^{-1}$ in the Pinheiros River).

Among the metals in the water samples, Lead concentrations were high, particularly in the Tietê River (maximum of $0.15 \mu \mathrm{g} . \mathrm{L}^{-1}$ ), as Chromium concentrations in the Pinheiros River (maximum of $0.31 \mathrm{mg} . \mathrm{L}^{-1}$ for both total and trivalent Chromium). Soluble Iron concentrations were higher in the Pinheiros River in comparison to the Tietê River (maximum of $14.3 \mathrm{mg} . \mathrm{L}^{-1}$

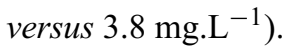

Thermotolerant Coliforms concentrations were similar in both rivers, since the highest values were about $10^{7}$ MPN.L ${ }^{-1}$. Enterovirus was detected in both rivers, but Cryptosporidium sp. and Giardia sp. were absent for all samples. Biochemical Oxygen Demand and Chemical Oxygen Demand were greater in the Tietê River (434 mg.L ${ }^{-1}$ and 860 mg.L ${ }^{-1}$ as maximum values, respectively). The highest concentrations of Total Phosphorus and Ammonia-Nitrogen, which are nutrients straightly related to the eutrophication, were $14.0 \mathrm{mg} . \mathrm{L}^{-1}$ (mean: $0.76 \mathrm{mg} . \mathrm{L}^{-1}$ ) and $99.0 \mathrm{mg} . \mathrm{L}^{-1}$ (mean: $23.3 \mathrm{mg} . \mathrm{L}^{-1}$ ) for the Tietê River, respectively, and 5.4 mg.. $\mathrm{L}^{-1}$ (mean: 0.54 mg. $\mathrm{L}^{-1}$ ) and 117 mg.L ${ }^{-1}$ (mean: 25.5 mg. $\mathrm{L}^{-1}$ ) for the Pinheiros River. Dissolved Oxygen concentrations and Turbidity values varied from $0.3 \mathrm{mg} . \mathrm{L}^{-1}$ to $7.3 \mathrm{mg} . \mathrm{L}^{-1}$ and 2 NTU to 107 NTU (Tietê River) and from $<0.1 \mathrm{mg} . \mathrm{L}^{-1}$ to $5.3 \mathrm{mg} . \mathrm{L}^{-1}$ and $5 \mathrm{NTU}$ to 217 NTU (Pinheiros River).

Concerning Case A ("exclusivity") variables, 11 variables were only detected in the Tietê River, including 9 organic compounds, 1 metal and 1 metalloid. On 
TABLE I

Results of the quantification of biological, chemical and physical variables that were detected in the Tietê and/or in Pinheiros Rivers (São Paulo State, Brazil) with their mean (Mean), minimum (Min) and maximum (Max) values, besides the coefficient of variation (C.V.) and the respective number of samples $(\mathrm{N})$ for each case.

\begin{tabular}{|c|c|c|c|c|c|}
\hline \multirow{2}{*}{ Variable } & \multicolumn{5}{|c|}{ Tietê River } \\
\hline & $\mathrm{N}$ & Mean & Min & Max & C.V. $(\%)$ \\
\hline 1.1-Dichloroethene & 15 & $<2.0 \mu \mathrm{g} . \mathrm{L}^{-1}$ & $<2.0 \mu \mathrm{g} . \mathrm{L}^{-1}$ & $13.0 \mu \mathrm{g} . \mathrm{L}^{-1}$ & 172.1 \\
\hline 1.2-Diphenylhydrazine & 11 & $0.05 \mu \mathrm{g} . \mathrm{L}^{-1}$ & $<0.05 \mu \mathrm{g} . \mathrm{L}^{-1}$ & $0.29 \mu \mathrm{g} . \mathrm{L}^{-1}$ & 162.8 \\
\hline $2,4-\mathrm{D}$ & 16 & $0.066 \mu$ g.L $\mathrm{L}^{-1}$ & $<0.0024 \mu \mathrm{g} . \mathrm{L}^{-1}$ & $0.874 \mu \mathrm{g} . \mathrm{L}^{-1}$ & 330.1 \\
\hline 2.4-Dichlorophenol & 17 & $0.7 \mu \mathrm{g} . \mathrm{L}^{-1}$ & $<0.003 \mu \mathrm{g} . \mathrm{L}^{-1}$ & $8.2 \mu \mathrm{g} . \mathrm{L}^{-1}$ & 287.3 \\
\hline 2.4-Dinitrotoluene & 2 & $0.1 \mu \mathrm{g} . \mathrm{L}^{-1}$ & $<0.1 \mu \mathrm{g} . \mathrm{L}^{-1}$ & $0.1 \mu \mathrm{g} . \mathrm{L}^{-1}$ & 33.2 \\
\hline $2.4 .5-\mathrm{T}$ & 17 & $0.2 \mu \mathrm{g} . \mathrm{L}^{-1}$ & $<0.0042 \mu \mathrm{g} . \mathrm{L}^{-1}$ & $3.0 \mu \mathrm{g} . \mathrm{L}^{-1}$ & 348.5 \\
\hline 2.4.6-Trichlorophenol & 17 & $0.009 \mu \mathrm{g} . \mathrm{L}^{-1}$ & $<0.005 \mu \mathrm{g} . \mathrm{L}^{-1}$ & $0.11 \mu \mathrm{g} . \mathrm{L}^{-1}$ & 293.9 \\
\hline 2-Chlorophenol & 2 & $0.1 \mu \mathrm{g} . \mathrm{L}^{-1}$ & $0.1 \mu \mathrm{g} . \mathrm{L}^{-1}$ & $0.1 \mu \mathrm{g} . \mathrm{L}^{-1}$ & - \\
\hline 4.4-DDD & 12 & $<0.0005 \mu \mathrm{g} . \mathrm{L}^{-1}$ & $<0.0005 \mu \mathrm{g} . \mathrm{L}^{-1}$ & $<0.0005 \mu \mathrm{g} . \mathrm{L}^{-1}$ & - \\
\hline 4.4-DDE & 12 & $0.001 \mu \mathrm{g} . \mathrm{L}^{-1}$ & $<0.0005 \mu \mathrm{g} . \mathrm{L}^{-1}$ & $0.005 \mu \mathrm{g} . \mathrm{L}^{-1}$ & 177.5 \\
\hline Acroleine & 10 & $1.6 \mu \mathrm{g} . \mathrm{L}^{-1}$ & $<0.5 \mu$ g. $\mathrm{L}^{-1}$ & $13 \mu \mathrm{g} . \mathrm{L}^{-1}$ & 252.5 \\
\hline Alachlor & 14 & $<0.0008 \mu \mathrm{g} . \mathrm{L}^{-1}$ & $<0.0008 \mu \mathrm{g} . \mathrm{L}^{-1}$ & $<0.0008 \mu \mathrm{g} . \mathrm{L}^{-1}$ & - \\
\hline Aldrin+Dieldrin & 16 & $<0.0007 \mu \mathrm{g} . \mathrm{L}^{-1}$ & $<0.0007 \mu \mathrm{g} . \mathrm{L}^{-1}$ & $<0.0007 \mu \mathrm{g} . \mathrm{L}^{-1}$ & - \\
\hline Alfa-BHC & 13 & $0.005 \mu \mathrm{g} . \mathrm{L}^{-1}$ & $<0.0008 \mu \mathrm{g} . \mathrm{L}^{-1}$ & $0.062 \mu \mathrm{g} . \mathrm{L}^{-1}$ & 332.5 \\
\hline Aluminum (soluble) & 18 & $0.42 \mathrm{mg} . \mathrm{L}^{-1}$ & $<0.1 \mathrm{mg} \cdot \mathrm{L}^{-1}$ & $2.3 \mathrm{mg} \cdot \mathrm{L}^{-1}$ & 120.0 \\
\hline Ammonia-nitrogen & 331 & $23.3 \mathrm{mg} . \mathrm{L}^{-1}$ & $0.2 \mathrm{mg} . \mathrm{L}^{-1}$ & $99.0 \mathrm{mg} . \mathrm{L}^{-1}$ & 61.2 \\
\hline Anthracene & 13 & $0.043 \mu \mathrm{g} . \mathrm{L}^{-1}$ & $<0.012 \mu \mathrm{g} . \mathrm{L}^{-1}$ & $0.486 \mu{\mathrm{g} . \mathrm{L}^{-1}}^{-1}$ & 310.2 \\
\hline Antimonium & 18 & $0.0046 \mu \mathrm{g} . \mathrm{L}^{-1}$ & $<0.0019 \mu \mathrm{g} . \mathrm{L}^{-1}$ & $15.0 \mu \mathrm{g} . \mathrm{L}^{-1}$ & 157.5 \\
\hline Apparent color & 457 & 304 C.U. & 3 C.U. & 956 C.U. & 61.6 \\
\hline Asbestos* & 13 & 4.4 Mf.L ${ }^{-1}$ & $<1.0{\mathrm{Mf} . \mathrm{L}^{-1}}^{-1}$ & $7.4 \mathrm{Mf} . \mathrm{L}^{-1}$ & 51.0 \\
\hline Barium & 18 & $0.045 \mathrm{mg} . \mathrm{L}^{-1}$ & $<0.01 \mathrm{mg.L^{-1 }}$ & $0.078 \mathrm{mg} . \mathrm{L}^{-1}$ & 47.9 \\
\hline Bentazon & 17 & $0.2 \mu \mathrm{g} . \mathrm{L}^{-1}$ & $<0.0042 \mu \mathrm{g} . \mathrm{L}^{-1}$ & $1.4 \mu \mathrm{g} . \mathrm{L}^{-1}$ & 206.2 \\
\hline Benz(a)Anthracene & 17 & $0.017 \mu \mathrm{g} . \mathrm{L}^{-1}$ & $<0.031 \mu \mathrm{g} . \mathrm{L}^{-1}$ & $0.04 \mu \mathrm{g} . \mathrm{L}^{-1}$ & 35.1 \\
\hline Biochemical Oxygen Demand & 455 & $121 \mathrm{mg} . \mathrm{L}^{-1}$ & $<2$ mg.L ${ }^{-1}$ & 434 mg.L ${ }^{-1}$ & 55.4 \\
\hline Bis (2-ethylhexyl)adipate & 10 & $0.5 \mu \mathrm{g} . \mathrm{L}^{-1}$ & $<0.1 \mu \mathrm{g} . \mathrm{L}^{-1}$ & $1.0 \mu \mathrm{g} . \mathrm{L}^{-1}$ & 31.3 \\
\hline Bis (2-ethylhexyl)ftalate & 13 & $1.0 \mu \mathrm{g} . \mathrm{L}^{-1}$ & $<1.0 \mu \mathrm{g} . \mathrm{L}^{-1}$ & $7.2 \mu \mathrm{g} . \mathrm{L}^{-1}$ & 182.6 \\
\hline Boron & 17 & 0.069 & $0.036 \mathrm{mg} . \mathrm{L}^{-1}$ & $0.200 \mathrm{mg} . \mathrm{L}^{-1}$ & 53.7 \\
\hline Bromates & 7 & $0.01 \mu \mathrm{g} . \mathrm{L}^{-1}$ & $<0.01 \mu \mathrm{g} . \mathrm{L}^{-1}$ & $0.04 \mu \mathrm{g} . \mathrm{L}^{-1}$ & 111.1 \\
\hline Cadmium & 17 & $<0.001{\mathrm{mg} . \mathrm{L}^{-1}}^{-1}$ & $<0.001{\mathrm{mg} . \mathrm{L}^{-1}}^{-1}$ & $0.001 \mathrm{mg} . \mathrm{L}^{-1}$ & - \\
\hline Carbofuran & 13 & $0.04 \mu \mathrm{g} . \mathrm{L}^{-1}$ & $<0.05 \mu$ g.L ${ }^{-1}$ & $0.31 \mu \mathrm{g} . \mathrm{L}^{-1}$ & 187.2 \\
\hline Chemical Oxygen Demand & 458 & $118 \mathrm{mg} . \mathrm{L}^{-1}$ & $12 \mathrm{mg} . \mathrm{L}^{-1}$ & 860 mg.L ${ }^{-1}$ & 60.3 \\
\hline Chlorides & 18 & $78 \mathrm{mg} . \mathrm{L}^{-1}$ & $40 \mathrm{mg} \cdot \mathrm{L}^{-1}$ & 132 mg.L 1 & 37.5 \\
\hline Chloroform & 18 & $<5.0 \mu \mathrm{g} . \mathrm{L}^{-1}$ & $<5.0 \mu \mathrm{g} . \mathrm{L}^{-1}$ & $13.0 \mu \mathrm{g} . \mathrm{L}^{-1}$ & - \\
\hline Chromium (total) & 18 & $0.02 \mathrm{mg} . \mathrm{L}^{-1}$ & $<0.005 \mathrm{mg} . \mathrm{L}^{-1}$ & $0.06 \mathrm{mg} . \mathrm{L}^{-1}$ & 95.9 \\
\hline Chromium (trivalent) & 18 & $0.016 \mathrm{mg} . \mathrm{L}^{-1}$ & $<0.005 \mathrm{mg} . \mathrm{L}^{-1}$ & $0.055 \mathrm{mg} . \mathrm{L}^{-1}$ & 104.0 \\
\hline Cis-1.2-Dichloroethene & 18 & $3.2 \mu \mathrm{g} . \mathrm{L}^{-1}$ & $<2.0 \mu \mathrm{g} . \mathrm{L}^{-1}$ & $3.4 \mu \mathrm{g} . \mathrm{L}^{-1}$ & 11.2 \\
\hline Cobalt & 18 & $0.005 \mathrm{mg} . \mathrm{L}^{-1}$ & $<0.005 \mathrm{mg} . \mathrm{L}^{-1}$ & $0.004 \mathrm{mg} . \mathrm{L}^{-1}$ & 107.8 \\
\hline
\end{tabular}


TABLE I (continuation)

\begin{tabular}{|c|c|c|c|c|c|}
\hline \multirow{2}{*}{ Variable } & \multicolumn{5}{|c|}{ Tietê River } \\
\hline & $\mathrm{N}$ & Mean & Min & Max & C.V. (\%) \\
\hline Condutivity & 353 & $544 \mu{\mathrm{S} . \mathrm{cm}^{-1}}^{-1}$ & $7 \mu \mathrm{S} . \mathrm{cm}^{-1}$ & $1,510 \mu{\mathrm{S} . \mathrm{cm}^{-1}}^{-1}$ & 27.1 \\
\hline Copper (total) & 17 & $0.030 \mathrm{mg} . \mathrm{L}^{-1}$ & $<0.005 \mathrm{mg} . \mathrm{L}^{-1}$ & $0.08 \mathrm{mg} . \mathrm{L}^{-1}$ & 85.1 \\
\hline Cyanide & 17 & $0.02 \mathrm{mg} . \mathrm{L}^{-1}$ & $<0.005 \mathrm{mg} . \mathrm{L}^{-1}$ & $0.06 \mathrm{mg} . \mathrm{L}^{-1}$ & 133.0 \\
\hline Detergents & 455 & $2.0 \mathrm{mg} . \mathrm{L}^{-1}$ & $<0.05 \mathrm{mg} . \mathrm{L}^{-1}$ & $36.0 \mathrm{mg} . \mathrm{L}^{-1}$ & 1,477 \\
\hline Dissolved oxygen & 213 & $1.9 \mathrm{mg} . \mathrm{L}^{-1}$ & $0.3 \mathrm{mg} . \mathrm{L}^{-1}$ & $7.3 \mathrm{mg} . \mathrm{L}^{-1}$ & 55.6 \\
\hline Endosulfan & 16 & $0.012 \mu \mathrm{g} . \mathrm{L}^{-1}$ & $<0.001 \mu \mathrm{g} . \mathrm{L}^{-1}$ & $0.162 \mu \mathrm{g} . \mathrm{L}^{-1}$ & 348.6 \\
\hline Endrin & 16 & $0.009 \mu \mathrm{g} . \mathrm{L}^{-1}$ & $<0.0005 \mu \mathrm{g} . \mathrm{L}^{-1}$ & $0.067 \mu \mathrm{g} . \mathrm{L}^{-1}$ & 226.5 \\
\hline Enterovirus & 64 & - & Absent & Present & - \\
\hline Ethylbenzene & 14 & $<2.0 \mu \mathrm{g} . \mathrm{L}^{-1}$ & $<2.0 \mu \mathrm{g} . \mathrm{L}^{-1}$ & $3.0 \mu \mathrm{g} . \mathrm{L}^{-1}$ & - \\
\hline Fluoranthene & 7 & $<0.03 \mu \mathrm{g} . \mathrm{L}^{-1}$ & $<0.03 \mu \mathrm{g} . \mathrm{L}^{-1}$ & $<0.03 \mu \mathrm{g} . \mathrm{L}^{-1}$ & - \\
\hline Hexachlorobutadiene & 14 & $<2.0 \mu \mathrm{g} . \mathrm{L}^{-1}$ & $<2.0 \mu \mathrm{g} . \mathrm{L}^{-1}$ & $<2.0 \mu \mathrm{g} . \mathrm{L}^{-1}$ & - \\
\hline Iron (soluble) & 303 & $0.3 \mathrm{mg} . \mathrm{L}^{-1}$ & $<0.05 \mathrm{mg} . \mathrm{L}^{-1}$ & $3.8 \mathrm{mg} . \mathrm{L}^{-1}$ & 136.5 \\
\hline Lead & 17 & $0.02 \mu \mathrm{g} . \mathrm{L}^{-1}$ & $<0.01 \mu \mathrm{g} . \mathrm{L}^{-1}$ & $0.15 \mu \mathrm{g} . \mathrm{L}^{-1}$ & 227.2 \\
\hline Manganese (soluble) & 302 & $0.1 \mathrm{mg} . \mathrm{L}^{-1}$ & $<0.05 \mathrm{mg} . \mathrm{L}^{-1}$ & $1.9 \mathrm{mg} . \mathrm{L}^{-1}$ & 81.1 \\
\hline Manganese (total) & 13 & $0.20 \mathrm{mg} . \mathrm{L}^{-1}$ & $0.08 \mathrm{mg} . \mathrm{L}^{-1}$ & $0.27 \mathrm{mg} . \mathrm{L}^{-1}$ & 36.4 \\
\hline Methylene Chloride & 18 & $9.2 \mu \mathrm{g} . \mathrm{L}^{-1}$ & $<2.0 \mu$ g. $\mathrm{L}^{-1}$ & $75.0 \mu \mathrm{g} . \mathrm{L}^{-1}$ & 201.4 \\
\hline Nickel & 11 & $<0.05 \mu \mathrm{g} . \mathrm{L}^{-1}$ & $<0.05 \mu \mathrm{g} . \mathrm{L}^{-1}$ & $0.09 \mu \mathrm{g} . \mathrm{L}^{-1}$ & - \\
\hline Nitrate & 16 & $1.0 \mathrm{mg} . \mathrm{L}^{-1}$ & $<0.1 \mathrm{mg} . \mathrm{L}^{-1}$ & $13.0 \mathrm{mg} . \mathrm{L}^{-1}$ & 303.0 \\
\hline Oxamyl & 9 & $<0.05 \mu \mathrm{g} . \mathrm{L}^{-}$ & $<0.05 \mu \mathrm{g} . \mathrm{L}^{-1}$ & $<0.05 \mu \mathrm{g} . \mathrm{L}^{-1}$ & - \\
\hline $\mathrm{pH}$ & 225 & 7.18 & 5.09 & 11.20 & 7.8 \\
\hline Phenols & 20 & $0.08 \mu \mathrm{g} . \mathrm{L}^{-1}$ & $<0.01 \mu \mathrm{g} . \mathrm{L}^{-1}$ & $0.53 \mu \mathrm{g} . \mathrm{L}^{-1}$ & 141.6 \\
\hline Phosphorus & 309 & $0.76 \mathrm{mg} . \mathrm{L}^{-1}$ & $<0.2 \mathrm{mg} . \mathrm{L}^{-1}$ & $14.0 \mathrm{mg} . \mathrm{L}^{-1}$ & 161.9 \\
\hline Sedimentable solids & 329 & $0.65 \mathrm{~mL}^{2} \mathrm{~L}^{-1}$ & $<0.1{\mathrm{~mL} . \mathrm{L}^{-1}}^{-1}$ & $15.0 \mathrm{~mL} . \mathrm{L}^{-1}$ & 176.5 \\
\hline Silver & 13 & $0.002 \mathrm{mg} . \mathrm{L}^{-1}$ & $<0.002 \mathrm{mg} . \mathrm{L}^{-1}$ & $0.007 \mathrm{mg} . \mathrm{L}^{-1}$ & 107.3 \\
\hline Temperature & 216 & $23^{\circ} \mathrm{C}\left(73.4^{\circ} \mathrm{F}\right)$ & $12^{\circ} \mathrm{C}\left(53.6^{\circ} \mathrm{F}\right)$ & $28^{\circ} \mathrm{C}\left(82.4^{\circ} \mathrm{F}\right)$ & 13.0 \\
\hline Tetrachloroethylene & 14 & $2.4 \mu \mathrm{g} . \mathrm{L}^{-1}$ & $<2.0 \mu \mathrm{g} . \mathrm{L}^{-1}$ & $14.0 \mu \mathrm{g} . \mathrm{L}^{-1}$ & 134.0 \\
\hline Thermotolerant coliforms & 347 & $1 \times 10^{6}$ MPN.L ${ }^{-1}$ & Absent & $13 \times 10^{6}$ MPN.L ${ }^{-1}$ & 189.6 \\
\hline Tin (total) & 17 & $0.10 \mathrm{mg} . \mathrm{L}^{-1}$ & $<0.02 \mathrm{mg} . \mathrm{L}^{-1}$ & $0.31 \mathrm{mg} . \mathrm{L}^{-1}$ & 87.2 \\
\hline Toluene & 14 & $46.6 \mu \mathrm{g} . \mathrm{L}^{-1}$ & $<4.0 \mu \mathrm{g} . \mathrm{L}^{-1}$ & $106.0 \mu \mathrm{g} . \mathrm{L}^{-1}$ & 93.8 \\
\hline Total dissolved solids & 136 & $283 \mathrm{mg} \cdot \mathrm{L}^{-1}$ & $<10 \mathrm{mg} \cdot \mathrm{L}^{-1}$ & $592 \mathrm{mg} . \mathrm{L}^{-1}$ & 33.8 \\
\hline Total suspended solids & 300 & $46 \mathrm{mg} . \mathrm{L}^{-1}$ & $<10 \mathrm{mg} \cdot \mathrm{L}^{-1}$ & $990 \mathrm{mg} . \mathrm{L}^{-1}$ & 183.0 \\
\hline Trichloroethylene & 14 & $<2.0 \mu{\mathrm{g} . \mathrm{L}^{-1}}^{-1}$ & $<2.0 \mu \mathrm{g} . \mathrm{L}^{-1}$ & $3.0 \mu \mathrm{g} . \mathrm{L}^{-1}$ & - \\
\hline Turbidity & 236 & $35 \mathrm{NTU}$ & $2 \mathrm{NTU}$ & 107 NTU & 64.3 \\
\hline Vanadium & 13 & $<0.01{\mathrm{mg} . \mathrm{L}^{-}}^{-}$ & $<0.01 \mathrm{mg} . \mathrm{L}^{-1}$ & $0.01 \mathrm{mg} . \mathrm{L}^{-1}$ & - \\
\hline Vinyl Chloride & 17 & $<2.0 \mu \mathrm{g} . \mathrm{L}^{-1}$ & $<2.0 \mu \mathrm{g} . \mathrm{L}^{-1}$ & $12.0 \mu \mathrm{g} . \mathrm{L}^{-1}$ & - \\
\hline Zinc & 13 & $0.15 \mathrm{mg} . \mathrm{L}^{-1}$ & $0.02 \mathrm{mg} . \mathrm{L}^{-1}$ & $0.34 \mathrm{mg} . \mathrm{L}^{-1}$ & 78.7 \\
\hline
\end{tabular}


TABLE I (continuation)

\begin{tabular}{|c|c|c|c|c|c|}
\hline \multirow{2}{*}{ Variable } & \multicolumn{5}{|c|}{ Pinheiros River } \\
\hline & $\mathrm{N}$ & Mean & Min & Max & C.V. $(\%)$ \\
\hline 1.1-Dichloroethene & 14 & $2.1 \mu \mathrm{g} . \mathrm{L}^{-1}$ & $<2.0 \mu \mathrm{g} . \mathrm{L}^{-1}$ & $17.0 \mu \mathrm{g} . \mathrm{L}^{-1}$ & 199.6 \\
\hline 1.2-Diphenylhydrazine & 8 & $<0.05 \mu \mathrm{g} . \mathrm{L}^{-1}$ & $<0.05 \mu \mathrm{g} . \mathrm{L}^{-1}$ & $<0.05 \mu \mathrm{g} . \mathrm{L}^{-1}$ & - \\
\hline $2,4-\mathrm{D}$ & 7 & $<0.0024 \mu \mathrm{g} . \mathrm{L}^{-1}$ & $<0.0024 \mu \mathrm{g} . \mathrm{L}^{-1}$ & $<0.0024 \mu \mathrm{g} . \mathrm{L}^{-1}$ & - \\
\hline 2.4-Dichlorophenol & 7 & $1.5 \mu \mathrm{g} . \mathrm{L}^{-1}$ & $<0.003 \mu \mathrm{g} . \mathrm{L}^{-1}$ & $10.8 \mu \mathrm{g} . \mathrm{L}^{-1}$ & 264.3 \\
\hline 2.4-Dinitrotoluene & 2 & $0.1 \mu \mathrm{g} . \mathrm{L}^{-1}$ & $<0.1 \mu \mathrm{g} . \mathrm{L}^{-1}$ & $0.1 \mu \mathrm{g} . \mathrm{L}^{-1}$ & 51.1 \\
\hline $2.4 .5-\mathrm{T}$ & 7 & $<0.0042 \mu \mathrm{g} . \mathrm{L}^{-1}$ & $<0.0042 \mu \mathrm{g} . \mathrm{L}^{-1}$ & $<0.0042 \mu \mathrm{g} . \mathrm{L}^{-1}$ & - \\
\hline 2.4.6-Trichlorophenol & 7 & $<0.005 \mu \mathrm{g} . \mathrm{L}^{-1}$ & $<0.005 \mu \mathrm{g} . \mathrm{L}^{-1}$ & $<0.005 \mu \mathrm{g} . \mathrm{L}^{-1}$ & - \\
\hline 2-Chlorophenol & 2 & $0.1 \mu \mathrm{g} . \mathrm{L}^{-1}$ & $0.1 \mu \mathrm{g} . \mathrm{L}^{-1}$ & $0.1 \mu \mathrm{g} . \mathrm{L}^{-1}$ & - \\
\hline 4.4-DDD & 14 & $0.004 \mu \mathrm{g} . \mathrm{L}^{-1}$ & $<0.0005 \mu \mathrm{g} . \mathrm{L}^{-1}$ & $0.053 \mu \mathrm{g} . \mathrm{L}^{-1}$ & 350.9 \\
\hline 4.4-DDE & 14 & $0.185 \mu \mathrm{g} . \mathrm{L}^{-1}$ & $<0.0005 \mu \mathrm{g} . \mathrm{L}^{-1}$ & $2.6 \mu \mathrm{g} . \mathrm{L}^{-1}$ & 373.7 \\
\hline Acroleine & 12 & $1.6 \mu \mathrm{g} . \mathrm{L}^{-1}$ & $<0.5 \mu \mathrm{g} . \mathrm{L}^{-1}$ & $13 \mu \mathrm{g} . \mathrm{L}^{-1}$ & 231.9 \\
\hline Alachlor & 14 & $0.0199 \mu \mathrm{g} . \mathrm{L}^{-1}$ & $<0.0008 \mu$ g.L $\mathrm{L}^{-1}$ & $0.075 \mu \mathrm{g} . \mathrm{L}^{-1}$ & 348.0 \\
\hline Aldrin+Dieldrin & 17 & $0.0018 \mu \mathrm{g} . \mathrm{L}^{-1}$ & $<0.0007 \mu \mathrm{g} . \mathrm{L}^{-1}$ & $0.02 \mu \mathrm{g} . \mathrm{L}^{-1}$ & 271.4 \\
\hline Alfa-BHC & 14 & $0.003 \mu \mathrm{g} . \mathrm{L}^{-1}$ & $<0.0008 \mu \mathrm{g} . \mathrm{L}^{-1}$ & $0.031 \mu \mathrm{g} . \mathrm{L}^{-1}$ & 308.2 \\
\hline Aluminum (soluble) & 17 & $0.24 \mathrm{mg} . \mathrm{L}^{-1}$ & $<0.1 \mathrm{mg} . \mathrm{L}^{-1}$ & $0.5 \mathrm{mg} . \mathrm{L}^{-1}$ & 51.5 \\
\hline Ammonia-nitrogen & 326 & $25.5 \mathrm{mg} . \mathrm{L}^{-1}$ & $0.5 \mathrm{mg} . \mathrm{L}^{-1}$ & $117.0 \mathrm{mg} . \mathrm{L}^{-1}$ & 56.2 \\
\hline Anthracene & 14 & $0.047 \mu \mathrm{g} . \mathrm{L}^{-1}$ & $<0.012 \mu{\mathrm{g} . \mathrm{L}^{-1}}^{-1}$ & $0.583 \mu \mathrm{g} . \mathrm{L}^{-1}$ & 326.6 \\
\hline Antimonium & 17 & $<0.0019 \mu$ g. $\mathrm{L}^{-1}$ & $<0.0019 \mu \mathrm{g} . \mathrm{L}^{-1}$ & $<0.0019 \mu$ g. $\mathrm{L}^{-1}$ & - \\
\hline Apparent color & 456 & 425 C.U. & 20 C.U. & 2,580 C.U. & 68.8 \\
\hline Asbestos* & 13 & $8.4{\mathrm{Mf} . \mathrm{L}^{-1}}^{-1}$ & $<1.0{\mathrm{Mf} . \mathrm{L}^{-1}}^{-1}$ & $50.9{\mathrm{Mf} . \mathrm{L}^{-1}}^{-1}$ & 156.4 \\
\hline Barium & 17 & $0.059 \mathrm{mg} . \mathrm{L}^{-1}$ & $<0.01 \mathrm{mg} . \mathrm{L}^{-1}$ & $0.14 \mathrm{mg} . \mathrm{L}^{-1}$ & 55.7 \\
\hline Bentazon & 8 & $0.6 \mu \mathrm{g} . \mathrm{L}^{-1}$ & $<0.0042 \mu \mathrm{g} . \mathrm{L}^{-1}$ & $4.9 \mu \mathrm{g} . \mathrm{L}^{-1}$ & 281.9 \\
\hline Benz(a)Anthracene & 8 & 0.019 & $<0.031 \mu{\mathrm{g} . \mathrm{L}^{-1}}^{-1}$ & $0.04 \mu \mathrm{g} . \mathrm{L}^{-1}$ & 46.7 \\
\hline Biochemical Oxygen Demand & 454 & 117 mg.L ${ }^{-1}$ & $<2{\mathrm{mg} . \mathrm{L}^{-1}}^{-1}$ & $271 \mathrm{mg} . \mathrm{L}^{-1}$ & 47.6 \\
\hline Bis (2-ethylhexyl)adipate & 12 & $0.5 \mu \mathrm{g} . \mathrm{L}^{-1}$ & $<0.1 \mu \mathrm{g} . \mathrm{L}^{-1}$ & $1.0 \mu \mathrm{g} . \mathrm{L}^{-1}$ & 5.5 \\
\hline Bis (2-ethylhexyl)ftalate & 14 & $2.2 \mu{\mathrm{g} . \mathrm{L}^{-1}}^{-1}$ & $<1.0 \mu \mathrm{g} . \mathrm{L}^{-1}$ & $12.0 \mu \mathrm{g} . \mathrm{L}^{-1}$ & 175.2 \\
\hline Boron & 17 & 0.042 & $<0.027{\mathrm{mg} . \mathrm{L}^{-1}}^{-1}$ & 0.094 mg.L 1 & 43.8 \\
\hline Bromates & 8 & $0.01 \mu \mathrm{g} . \mathrm{L}^{-1}$ & $<0.01 \mu \mathrm{g} . \mathrm{L}^{-1}$ & $0.04 \mu \mathrm{g} . \mathrm{L}^{-1}$ & 113.1 \\
\hline Cadmium & 16 & $<0.001 \mathrm{mg} . \mathrm{L}^{-1}$ & $<0.001{\mathrm{mg} . \mathrm{L}^{-1}}^{-1}$ & $<0.001{\mathrm{mg} . \mathrm{L}^{-1}}^{-1}$ & - \\
\hline Carbofuran & 13 & $0.12 \mu \mathrm{g} . \mathrm{L}^{-1}$ & $<0.05 \mu \mathrm{g} . \mathrm{L}^{-1}$ & $0.76 \mu \mathrm{g} . \mathrm{L}^{-1}$ & 187.6 \\
\hline Chemical Oxygen Demand & 457 & $120 \mathrm{mg} . \mathrm{L}^{-1}$ & $20 \mathrm{mg} . \mathrm{L}^{-1}$ & 614 mg. $\mathrm{L}^{-1}$ & 49.1 \\
\hline Chlorides & 17 & $58 \mathrm{mg} . \mathrm{L}^{-1}$ & $30 \mathrm{mg} \cdot \mathrm{L}^{-1}$ & $116 \mathrm{mg} . \mathrm{L}^{-1}$ & 46.7 \\
\hline Chloroform & 17 & $6.6 \mu \mathrm{g} . \mathrm{L}^{-1}$ & $<5.0 \mu \mathrm{g} . \mathrm{L}^{-1}$ & $43.0 \mu \mathrm{g} . \mathrm{L}^{-1}$ & 150.1 \\
\hline Chromium (total) & 17 & $0.02 \mathrm{mg} . \mathrm{L}^{-1}$ & $<0.005 \mathrm{mg} . \mathrm{L}^{-1}$ & $0.31 \mathrm{mg} . \mathrm{L}^{-1}$ & 316.6 \\
\hline Chromium (trivalent) & 17 & $0.023 \mathrm{mg} . \mathrm{L}^{-1}$ & $<0.005{\mathrm{mg} . \mathrm{L}^{-1}}^{-1}$ & $0.31 \mathrm{mg} . \mathrm{L}^{-1}$ & 329.2 \\
\hline Cis-1.2-Dichloroethene & 17 & $<2.0 \mu \mathrm{g} . \mathrm{L}^{-1}$ & $<2.0 \mu \mathrm{g} . \mathrm{L}^{-1}$ & $<2.0 \mu \mathrm{g} . \mathrm{L}^{-1}$ & - \\
\hline Cobalt & 16 & $0.005 \mathrm{mg} . \mathrm{L}^{-1}$ & $<0.005 \mathrm{mg} . \mathrm{L}^{-1}$ & $0.006 \mathrm{mg} . \mathrm{L}^{-1}$ & 104.2 \\
\hline
\end{tabular}


TABLE I (continuation)

\begin{tabular}{|c|c|c|c|c|c|}
\hline \multirow{2}{*}{ Variable } & \multicolumn{5}{|c|}{ Pinheiros River } \\
\hline & $\mathrm{N}$ & Mean & Min & Max & C.V. $(\%)$ \\
\hline Condutivity & 454 & $500 \mu$ S.cm $^{-1}$ & $4 \mu \mathrm{S} . \mathrm{cm}^{-1}$ & $1,440 \mu{\mathrm{S} . \mathrm{cm}^{-1}}^{-1}$ & 33.1 \\
\hline Copper (total) & 16 & $0.016 \mathrm{mg} . \mathrm{L}^{-1}$ & $<0.005 \mathrm{mg} . \mathrm{L}^{-1}$ & $0.05 \mathrm{mg} . \mathrm{L}^{-1}$ & 85.8 \\
\hline Cyanide & 17 & $0.01 \mathrm{mg} . \mathrm{L}^{-1}$ & $<0.005 \mathrm{mg} . \mathrm{L}^{-1}$ & $0.035 \mathrm{mg} . \mathrm{L}^{-1}$ & 116.8 \\
\hline Detergents & 258 & $1.9 \mathrm{mg} . \mathrm{L}^{-1}$ & $<0.05 \mathrm{mg} . \mathrm{L}^{-1}$ & $11 \mathrm{mg} . \mathrm{L}^{-1}$ & 94.1 \\
\hline Dissolved oxygen & 303 & $1.6 \mathrm{mg} . \mathrm{L}^{-1}$ & $<0.1 \mathrm{mg} . \mathrm{L}^{-1}$ & $5.3 \mathrm{mg} . \mathrm{L}^{-1}$ & 63.8 \\
\hline Endosulfan & 8 & $<0.001 \mu \mathrm{g} . \mathrm{L}^{-1}$ & $<0.001 \mu \mathrm{g} . \mathrm{L}^{-1}$ & $<0.001 \mu \mathrm{g} . \mathrm{L}^{-1}$ & - \\
\hline Endrin & 8 & $<0.0005 \mu \mathrm{g} . \mathrm{L}^{-1}$ & $<0.0005 \mu \mathrm{g} . \mathrm{L}^{-1}$ & $<0.0005 \mu \mathrm{g} . \mathrm{L}^{-1}$ & - \\
\hline Enterovirus & 56 & - & Absent & Present & - \\
\hline Ethylbenzene & 13 & $<2.0 \mu \mathrm{g} . \mathrm{L}^{-1}$ & $<2.0 \mu \mathrm{g} . \mathrm{L}^{-1}$ & $2.5 \mu \mathrm{g} . \mathrm{L}^{-1}$ & - \\
\hline Fluoranthene & 6 & $0.03 \mu \mathrm{g} . \mathrm{L}^{-1}$ & $<0.03 \mu \mathrm{g} . \mathrm{L}^{-1}$ & $0.08 \mu \mathrm{g} . \mathrm{L}^{-1}$ & 103.0 \\
\hline Hexachlorobutadiene & 13 & $<2.0 \mu \mathrm{g} . \mathrm{L}^{-1}$ & $<2.0 \mu \mathrm{g} . \mathrm{L}^{-1}$ & $6.0 \mu \mathrm{g} . \mathrm{L}^{-1}$ & - \\
\hline Iron (soluble) & 103 & $2.1 \mathrm{mg} . \mathrm{L}^{-1}$ & $<0.05 \mathrm{mg} . \mathrm{L}^{-1}$ & $14.3 \mathrm{mg} . \mathrm{L}^{-1}$ & 160.0 \\
\hline Lead & 16 & $<0.01 \mu \mathrm{g} . \mathrm{L}^{-1}$ & $<0.01 \mu \mathrm{g} . \mathrm{L}^{-1}$ & $0.032 \mu$ g. $\mathrm{L}^{-1}$ & - \\
\hline Manganese (soluble) & 102 & $0.2 \mathrm{mg} . \mathrm{L}^{-1}$ & $<0.05 \mathrm{mg} . \mathrm{L}^{-1}$ & $2.2 \mathrm{mg} . \mathrm{L}^{-1}$ & 82.4 \\
\hline Manganese (total) & 12 & $0.23 \mathrm{mg} . \mathrm{L}^{-1}$ & $0.1 \mathrm{mg} . \mathrm{L}^{-1}$ & $0.63 \mathrm{mg} . \mathrm{L}^{-1}$ & 65.9 \\
\hline Methylene Chloride & 17 & $2.9 \mu \mathrm{g} . \mathrm{L}^{-1}$ & $<2.0 \mu \mathrm{g} . \mathrm{L}^{-1}$ & $15.0 \mu \mathrm{g} . \mathrm{L}^{-1}$ & 141.8 \\
\hline Nickel & 12 & $<0.05 \mu \mathrm{g} . \mathrm{L}^{-1}$ & $<0.05 \mu \mathrm{g} . \mathrm{L}^{-1}$ & $0.03 \mu \mathrm{g} . \mathrm{L}^{-1}$ & - \\
\hline Nitrate & 15 & $0.1 \mathrm{mg} . \mathrm{L}^{-1}$ & $<0.1 \mathrm{mg} . \mathrm{L}^{-1}$ & $0.5 \mathrm{mg} . \mathrm{L}^{-1}$ & 110.9 \\
\hline Oxamyl & 9 & $0.1 \mu \mathrm{g} . \mathrm{L}^{-1}$ & $<0.05 \mu \mathrm{g} . \mathrm{L}^{-1}$ & $0.9 \mu \mathrm{g} . \mathrm{L}^{-1}$ & 263.7 \\
\hline $\mathrm{pH}$ & 316 & 7.10 & 3.00 & 10.70 & 7.4 \\
\hline Phenols & 9 & $0.04 \mu \mathrm{g} . \mathrm{L}^{-1}$ & $<0.01 \mu \mathrm{g} . \mathrm{L}^{-1}$ & $0.16 \mu \mathrm{g} . \mathrm{L}^{-1}$ & 150.5 \\
\hline Phosphorus & 299 & $0.59 \mathrm{mg} . \mathrm{L}^{-1}$ & $<0.2 \mathrm{mg} . \mathrm{L}^{-1}$ & $5.4 \mathrm{mg} . \mathrm{L}^{-1}$ & 128.6 \\
\hline Sedimentable solids & 325 & $1.1 \mathrm{~mL} . \mathrm{L}^{-1}$ & $<0.1{\mathrm{~mL} . \mathrm{L}^{-1}}^{-1}$ & $10.1 \mathrm{~mL} \mathrm{~L}^{-1}$ & 787.2 \\
\hline Silver & 12 & $0.002 \mathrm{mg} . \mathrm{L}^{-1}$ & $<0.002 \mathrm{mg} . \mathrm{L}^{-1}$ & $0.003 \mathrm{mg} . \mathrm{L}^{-1}$ & 111.5 \\
\hline Temperature & 307 & $23^{\circ} \mathrm{C}\left(73.4^{\circ} \mathrm{F}\right)$ & $16^{\circ} \mathrm{C}\left(60.8^{\circ} \mathrm{F}\right)$ & $33^{\circ} \mathrm{C}\left(91.4^{\circ} \mathrm{F}\right)$ & 12.4 \\
\hline Tetrachloroethylene & 13 & $<2.0 \mu \mathrm{g} . \mathrm{L}^{-1}$ & $<2.0 \mu \mathrm{g} . \mathrm{L}^{-1}$ & $<2.0 \mu \mathrm{g} . \mathrm{L}^{-1}$ & - \\
\hline Thermotolerant coliforms & 258 & $3 \times 10^{5}{\mathrm{MPN} . \mathrm{L}^{-1}}^{-1}$ & Absent & $12 \times 10^{6}$ MPN.L $^{-1}$ & 296.3 \\
\hline Tin (total) & 17 & $0.10 \mathrm{mg} . \mathrm{L}^{-1}$ & $<0.02 \mathrm{mg} . \mathrm{L}^{-1}$ & $0.51 \mathrm{mg} . \mathrm{L}^{-1}$ & 119.0 \\
\hline Toluene & 13 & $85.4 \mu \mathrm{g} . \mathrm{L}^{-1}$ & $<4.0 \mu \mathrm{g} . \mathrm{L}^{-1}$ & $215.0 \mu \mathrm{g} . \mathrm{L}^{-1}$ & 77.0 \\
\hline Total dissolved solids & 99 & $245 \mathrm{mg} . \mathrm{L}^{-1}$ & $86 \mathrm{mg} . \mathrm{L}^{-1}$ & $455 \mathrm{mg} . \mathrm{L}^{-1}$ & 36.7 \\
\hline Total suspended solids & 297 & $37 \mathrm{mg} . \mathrm{L}^{-1}$ & $<10 \mathrm{mg} \cdot \mathrm{L}^{-1}$ & $340 \mathrm{mg} . \mathrm{L}^{-1}$ & 123.3 \\
\hline Trichloroethylene & 13 & $<2.0 \mu \mathrm{g} . \mathrm{L}^{-1}$ & $<2.0 \mu \mathrm{g} . \mathrm{L}^{-1}$ & $3.0 \mu \mathrm{g} . \mathrm{L}^{-1}$ & - \\
\hline Turbidity & 326 & $56 \mathrm{NTU}$ & $5 \mathrm{NTU}$ & 217 NTU & 70.1 \\
\hline Vanadium & 12 & $<0.01{\mathrm{mg} . \mathrm{L}^{-}}^{-}$ & $<0.01 \mathrm{mg} . \mathrm{L}^{-1}$ & 0.03 mg.L ${ }^{-1}$ & - \\
\hline Vinyl Chloride & 15 & $<2.0 \mu \mathrm{g} . \mathrm{L}^{-1}$ & $<2.0 \mu \mathrm{g} . \mathrm{L}^{-1}$ & $<2.0 \mu$ g. $\mathrm{L}^{-1}$ & - \\
\hline Zinc & 11 & $0.07 \mathrm{mg} . \mathrm{L}^{-1}$ & $0.04 \mathrm{mg} . \mathrm{L}^{-1}$ & $0.16 \mathrm{mg} . \mathrm{L}^{-1}$ & 64.3 \\
\hline
\end{tabular}

* Mf. $\mathrm{L}^{-1}$ : Millions of fibers per Liter. 
the other hand, 6 substances were exclusively detected in the Pinheiros River, being all of them organic compounds (Table II).

Five variables exclusively detected in the Tietê River (2.4-D, 2.4.5-T, 2.4.6-Trichlorophenol, Endosulfan and Endrin) are organic compounds normally used in agriculture to control plagues, like weeds, insects and fungus, although agricultural activities are not preponderant in the Tietê sub-basin (there are only some vegetable belts in the upper part of the basin). All these pesticides present chlorine in their molecular structure. Four other organic substances, which were also only observed in the Tietê River, may be related with industrial activities that take place in the respective subbasin: 1.2-Diphenylhydrazine, Cis-1.2-Dichloroethene, Vinyl Chloride and Tetrachloroethylene. The degradation of 1.2-Diphenylhydrazine promotes the formation of several other products that may be associated with the slow disappearance of its overall toxicity (Muneer et al. 2002). This cited study ratified that this substance presents a high level of toxicity, even through its degradation products.

Cis-1.2-Dichloroethene and Vinyl Chloride in turn are recognized as biodegradation products of Trichloroethylene and Tetrachloroetylene (Bradley et al. 1998). Tetrachloroetylene sources to the Tietê River may include dry cleaning establishments and metal degreasing activities. The anoxic conditions found in the Tietê River (the mean and minimum concentrations of dissolved oxygen were $1.9 \mathrm{mg} . \mathrm{L}^{-1}$ and $0.3 \mathrm{mg} . \mathrm{L}^{-1}$, respectively) were probably able to stimulate the degradation of Tetrachloroetylene to Dichloroethene and Vinyl Chloride through anaerobic reductive dechlorination, as verified by other researchers in lab-scale experiments (Bradley et al. 2008, Duhamel et al. 2002). Cadmium and Antimonium concentrations in the Tietê River may be associated with the clandestine discharges of metallurgical units, which are common in the surrounding area, and with the effluents of oil-handling industry.

When it comes to the substances exclusively detected in the Pinheiros River, all of them are organic compounds, four of which are used to decrease plagues infestation (three are chlorinated). Agricultural activities in the Pinheiros River sub-basin are almost inexistent. Therefore, it is assumed that a possible source of these compounds may be the application of insecticides in the river margins to eliminate mosquitoes and other vectors, mainly organophosphate compounds like Themephos and Cipermetrine (aleatory application) and Betacyfluthrin (daily application), according to Morais et al. (2007). Some authors consider the Pinheiros River as a large urban breeding of the mosquito Culex quinquefasciatus and state that this situation was aggravated since the pumping of its water to Billings Reservoir was interrupted (Bracco et al. 1997, Morais et al. 2006, Andrade et al. 2007, Silva-Filha et al. 2008).

In the case of Alachlor, this herbicide has relatively rapid transformation rates and, consequently, its overall occurrence and concentration may be underestimated without data on the degradation products, as verified by Kolpin et al. (1998) for North-American groundwater. Fluoranthene occurrence may be related with effluents of coal combustion, vehicular exhaust, tire degradation and lubricating oils industry (Manoli et al. 2000), which are widespread in the Pinheiros River sub-basin. Sealed areas, such as streets and other urban sites, are successively re-enriched by PAHs (Polycyclic Aromatic Hydrocarbons) like Fluoranthene, for instance. The input of this substance into the aquatic systems is controlled by the location and activation of particle sources and, consequently, urban runoff plays an important role over these processes (Krein and Schorer 2000, Hwang and Foster 2006). Hexachlorobutadiene in turn is also related with industrial activities. This aliphatic compound is extremely volatile and some recent researches have been linking hexachlorobutadiene exposure with possible carcinogenic effects to human (Tchounwou et al. 1998, Green et al. 2003, Staples et al. 2003, Juang et al. 2009).

Table III presents the variables that were placed in Case B ("presence") category, with "TIE" meaning that the highest value was found in the Tietê River and "PIN" denoting the same for the Pinheiros River.

In synthesis, 56 variables were detected in both rivers, but the highest values or concentrations of 26 were found in the Tietê River and 22 in the Pinheiros River. Case B-TIE variables included diverse groups: 5 organic compounds, 2 nutrients, 6 physical variables, 7 metals, 1 non-metallic substance, 2 ions and 1 biological variable, besides $\mathrm{BOD}$ and $\mathrm{COD}$, which are indic- 
TABLE II

Case A ("exclusivity") variables, which were exclusively detected either in the Tietê or in Pinheiros River, including some information about each one.

\begin{tabular}{|c|c|}
\hline Variable & Information \\
\hline \multicolumn{2}{|r|}{ Tietê River } \\
\hline 1.2-Diphenylhydrazine & $\begin{array}{l}\text { Organic compound used to produce some dyes and } \\
\text { to make certain medicines }\end{array}$ \\
\hline 2.4-D & Organic compound used as herbicide \\
\hline 2.4.5-T & Organic compound used as herbicide \\
\hline 2.4.6-Trichlorophenol & Organic compound used as fungicide \\
\hline Antimonium & $\begin{array}{l}\text { Metalloid used for metal blending and as a } \\
\text { component for storage batteries }\end{array}$ \\
\hline Cadmium & Metal used for batteries manufacture \\
\hline Cis-1.2-Dichloroethene & Organic compound used in the manufacture of solvents \\
\hline Endosulfan & Organic compound used as insecticide and fungicide \\
\hline Endrin & Organic compound used as insecticide \\
\hline Tetrachloroethylene & Organic compound used for dry cleaning and metal degreasing \\
\hline Vinyl Chloride & $\begin{array}{l}\text { Organic compound whose polymerization produces PVC, } \\
\text { a thermoplastic resin that is largely used worldwide }\end{array}$ \\
\hline \multicolumn{2}{|r|}{ Pinheiros River } \\
\hline 4.4-DDD & Organic compound used as insecticide \\
\hline Alachlor & Organic compound used as herbicide \\
\hline Aldrin+Dieldrin & Organic compound used as insecticide \\
\hline Fluoranthene & Organic compound, usually a byproduct of combustion process \\
\hline Hexachloro-butadiene & $\begin{array}{l}\text { Organic compound, usually a byproduct of industrial plants } \\
\text { that deal with hydrochloric acid }\end{array}$ \\
\hline Oxamyl & Organic compound used as nematocide \\
\hline
\end{tabular}

References: ATSDR (1999), PANNA (2009), TOXMAP (2009).

ators of degradable organic matter by biochemical or chemical processes, respectively. When it comes to Case B-PIN variables, the distribution was: 9 organic compounds, 1 nutrient, 3 physical variables and 8 metals, besides Asbestos. The Tietê River water presented the highest values of BOD (Biochemical Oxygen Demand), Conductivity, Detergents, Phosphorus, Solids (Sedimentable, Dissolved and Suspended) and Thermotolerant Coliforms, which are straightly related to organic matter pollution derived from domestic wastewater. Chloroform concentrations were high (reaching $43.0 \mu \mathrm{g} . \mathrm{L}^{-1}$ in the Pinheiros River and $13.0 \mu \mathrm{g} . \mathrm{L}^{-1}$ in the Tietê River), when compared to the mean concentration for European rivers, for instance, which is about $0.5 \mu \mathrm{g} . \mathrm{L}^{-1}$ (McCulloch 2003). The same happens for Aluminum (maximum of $2.3 \mathrm{mg} . \mathrm{L}^{-1}$ in the Tietê River), Chromium (maximum of $0.31 \mathrm{mg} . \mathrm{L}^{-1}$ in the Pinheiros River) and Iron (maximum of $14.3 \mathrm{mg} . \mathrm{L}^{-1}$ in the Pinheiros River).

When we assessed the temporal component of the monitoring program, the Analysis of Variance (ANOVA) with variables from Case A and B (altogether 73 variables) suggested that only 11 variables were considered statistically different comparing the rainy and dry periods (Table IV). These variables were directly influenced by the rainfall in the urban area, e.g. Total suspended solids, which were higher in the rainy months. On the other hand, Apparent color, Chemical Oxygen Demand and Conductivity, which are variables that may be related with industrial effluents, presented 
TABLE III

Case B ("presence") variables, which were detected both in the Tietê and Pinheiros Rivers.

\begin{tabular}{|c|c|c|c|}
\hline Variable & $\begin{array}{c}\text { Highest } \\
\text { concentration/value }\end{array}$ & Variable & $\begin{array}{c}\text { Highest } \\
\text { concentration/value }\end{array}$ \\
\hline 1.1-Dichloroethene & PIN & Cyanide & TIE \\
\hline 2.4-Dichlorophenol & PIN & Detergents & $\mathrm{TIE}$ \\
\hline 2.4-Dinitrotoluene & $\mathrm{TIE}=\mathrm{PIN}$ & Dissolved Oxygen & TIE \\
\hline 2-Chlorophenol & TIE $=$ PIN & Enterovirus & TIE $=$ PIN \\
\hline 4.4-DDE & PIN & Ethylbenzene & TIE \\
\hline Acroleine & $\mathrm{TIE}=\mathrm{PIN}$ & Iron (soluble) & PIN \\
\hline Alfa-BHC & TIE & Lead & TIE \\
\hline Aluminum (soluble) & TIE & $\mathrm{pH}$ & TIE \\
\hline Ammonia-nitrogen & PIN & Phenols & TIE \\
\hline Anthracene & PIN & Phosphorus & TIE \\
\hline Apparent color & PIN & Manganese (soluble) & PIN \\
\hline Asbestos & PIN & Manganese (total) & TIE \\
\hline Barium & PIN & Methylene Chloride & TIE \\
\hline Bentazon & PIN & Nickel & TIE \\
\hline Benz(a)Anthracene & $\mathrm{TIE}=\mathrm{PIN}$ & Nitrate & TIE \\
\hline Biochemical Oxygen Demand & TIE & Sedimentable solids & TIE \\
\hline Bis (2-ethylhexyl)adipate & $\mathrm{TIE}=\mathrm{PIN}$ & Silver & TIE \\
\hline Bis (2-ethylhexyl)ftalate & PIN & Temperature & PIN \\
\hline Boron & TIE & Thermotolerant Coliforms & TIE \\
\hline Bromates & $\mathrm{TIE}=\mathrm{PIN}$ & Tin (total) & PIN \\
\hline Carbofuran & PIN & Toluene & PIN \\
\hline Chemical Oxygen Demand & TIE & Total dissolved solids & TIE \\
\hline Chlorides & TIE & Total suspended solids & TIE \\
\hline Chloroform & PIN & Trichloroethylene & TIE $=$ PIN \\
\hline Chromium (total) & PIN & Turbidity & PIN \\
\hline Chromium (trivalent) & PIN & Vanadium & PIN \\
\hline Cobalt & PIN & Zinc & TIE \\
\hline Copper (total) & TIE & \multirow{2}{*}{\multicolumn{2}{|c|}{$\begin{array}{l}\text { TOTAL: TIE ( } 26 \text { variables), } \\
\text { PIN ( } 22 \text { variables), TIE }=\text { PIN ( } 8 \text { variables) }\end{array}$}} \\
\hline Condutivity & TIE & & \\
\hline
\end{tabular}

their highest values in the dry season, possibly as a consequence of lower river flows and smaller capacity of dilution.

The remaining 61 variables that were not aforementioned (51 organic compounds, 8 metals and 2 biological variables) were placed in Case C (“total absence") since they were not detected in any sample, considering the detection limit: 1.1.1-Trichloroethane, 1.1.2.2Tetrachloroethane, 1.2.4-Trichlorobenzene, 1.2-Dichlorobenzene, 1.2-Dichloroethane, 1.2-Dichloropropane, 1.2-trans-Dichloroethene, 1.3-Dichlorobenzene, 1.3-Di- chloropropene, 1.4-Dichlorobenzene, 2.4-Dinitrophenol, 2.4.5-TP, 2.4-Dimetylphenol, 2-Methyl-4.6-Dinitrophenol, 3.3-Dichlorobenzidine, 4.4-DDT, Acenaphtene, Acrylamide, Aldicarb, Arsene, Atrazine, Benz(a)Pyrene, Benzene, Benzo(b)Fluoranthene, Benzo(k)Fluoranthene, Beryllium, Beta-BHC, Bis (2-Chloroethylether), Bis (2-Chloroisopropyl-ether), Chlordane, Chlorobenzene, Chromium (hexavalent), Chrysene, Copper (soluble), Cryptosporidium sp., Dalapon, Demeton, Dibenz(a,h)anthracene, Dieldrin, Dinoseb, Endosulfan sulfate, Fluorene, Giardia sp., Guthion, Heptachloro, 
TABLE IV

List of variables that presented statistically significant differences during the dry and rainy periods in the Tietê and/or Pinheiros Rivers. Observation: p $^{*}$ indicates statistical significance.

\begin{tabular}{l|c|c|l}
\multicolumn{1}{c|}{ Variable } & Tietê River & Pinheiros River & \multicolumn{1}{c}{ Observation } \\
\hline Apparent color & $\mathrm{p}^{*}<0.05$ & $\mathrm{p}^{*}<0.05$ & Higher in the dry period \\
Chemical Oxygen Demand & $\mathrm{p}^{*}<0.05$ & $\mathrm{p}^{*}<0.05$ & Higher in the dry period \\
Conductivity & $\mathrm{p}^{*}<0.05$ & $\mathrm{p}^{*}<0.05$ & Higher in the dry period \\
Dissolved oxygen & $\mathrm{p}^{*}<0.05$ & $\mathrm{p}>0.05$ (NSS) & Higher in the dry period \\
$\mathrm{pH}$ & $\mathrm{p}^{*}<0.05$ & $\mathrm{p}^{*}<0.05$ & Higher in the rainy period \\
Phosphorus & $\mathrm{p}^{*}<0.05$ & $\mathrm{p}^{*}<0.05$ & Higher in the rainy period \\
Sedimentable solids & $\mathrm{p}^{*}<0.05$ & $\mathrm{p}>0.05$ (NSS) & Higher in the rainy period \\
Temperature & $\mathrm{p}^{*}<0.05$ & $\mathrm{p}^{*}<0.05$ & Higher in the rainy period \\
Total dissolved solids & $\mathrm{p}^{*}<0.05$ & $\mathrm{p}>0.05$ (NSS) & Higher in the dry period \\
Total suspended solids & $\mathrm{p}^{*}<0.05$ & $\mathrm{p}^{*}<0.05$ & Higher in the rainy period \\
Turbidity & $\mathrm{p}^{*}<0.05$ & $\mathrm{p}^{*}<0.05$ & Higher in the dry period \\
\hline
\end{tabular}

NSS: the difference was not statistically significant.

Hexachlorobenzene, Hexachloroethane, Indeno (1.2.3cd) pyrene, Lindane, Lithium, Malathion, Mercury, Methyl-bromide, Molinate, Pentachlorophenol, Polychlorinated biphenyls, Selenium, Styrene, Toxaphene, Uranium and Xylene.

Among the metals allocated in Case C, we highlight Chromium (hexavalent), Lithium, Mercury and Uranium, which were absent. They are toxic heavy metals that may exert detrimental effects not only on human health but also on the whole environment (Davydova 2005). Cryptosporidium sp. and Giardia sp. were also absent for all samples of the Tietê and Pinheiros Rivers. Giardia sp. causes an intestinal illness called giardiasis or "beaver fever" and Cryptosporidium sp. is responsible for a similar illness called cryptosporidiosis, which is characterized by gastroenteritis (Franco et al. 2001, Hachich 2002, Heller et al. 2004).

A considerable level of differences was found for the studied urban rivers, not only between the aquatic systems themselves, but also considering the same river in distinct periods of the hydrologic cycle (i.e. dry and wet seasons). Case A variables particularly represent a key information, since the Tietê River and the Pinheiros River presented some exclusive occurrences. Therefore, the imperative is to analyze each river separately and to establish individualized recovery plans for each one, taking into account, besides the seasonal fluctuation, the fact that all exclusive variables of the Pinheiros River are organic compounds and, on the other hand, the exclusive water variables in the Tietê River included, besides some organic substances, a metal and a metalloid.

\section{CONCLUSIONS}

The water quality monitoring of the Tietê and Pinheiros Rivers (Metropolitan Region of São Paulo), through an intense program that performed the quantification of biological, chemical and physical variables for about seventeen months (August, 2007 to December, 2008) enabled the authors to conclude that:

i. It is not advisable to adopt the same management program of water resources or control procedures for the sub-basins of both rivers. The results showed that, despite the fact that they are located in the same basin, the contribution of the drainage sub-basin area was relevant and determined some exclusive water variables occurrences for these urban aquatic systems;

ii. The temporal variation of the results presented a significant importance for only eleven variables, among the seventy three that were assessed. These variables were influenced by rainfall events and different seasonal precipitation patterns. Some of these variables were statistically different within the dry and rainy months only for the Tietê River (e.g. Dissolved Oxygen, Sedimentable solids and 
Total dissolved solids), which suggested that the temporal component is even more important for the former aquatic system and that this river is possibly more susceptible to the urban runoff;

iii. In general, the Tietê River seemed to be more affected by domestic wastewater in comparison to the Pinheiros River, since high concentrations of Phosphorus, Ammonia-Nitrogen, BOD and detergents were observed in the former river. Nevertheless, by analyzing the variables of exclusive occurrence, it was possible to find out that the Tietê River is also submitted to impacts from industry, from the inappropriate disposal of batteries and solvents and from dry cleaning and metal degreasing activities;

iv. The Pinheiros River in turn also presented some exclusive occurrences, mainly organochlorinated compounds, although the Pinheiros sub-basin has almost none agricultural activities. Therefore, these occurrences were associated with the application of insecticides on the river margins to reduce mosquitoes infestation, which is a current practice in the area. Besides, the carcinogenic substance was exclusively detected in the water of this river, characterizing a public health concern;

$\mathrm{v}$. The rivers are severely polluted by a great number of substances, with diverse origins from the respective sub-basin. Particularly, the concentrations of Aluminum, Ammonia-Nitrogen, Chloroform, Cyanide, Detergents, Phosphorus and Solids were high in both rivers, which may provide significant risks to the water quality, to the aquatic systems balance and to the public health as well.

\section{ACKNOWLEDGMENTS}

We wish to express our sincere thanks to two anonymous reviewers for their interesting suggestions for improving the manuscript and to Fabiana Rodrigues Costa Nunes for reviewing the English writing of the paper.

\section{RESUMO}

O gerenciamento dos recursos hídricos urbanos desempenha um papel importante para os países em desenvolvimento. Os rios Tietê e Pinheiros (São Paulo, Brasil) são afetados por efluentes domésticos e industriais e pela poluição difusa. Esta pesquisa teve como objetivo quantificar 134 variáveis da água dos rios Tietê e Pinheiros (aproximadamente 7.200 e 6.600 análises, respectivamente) de Agosto de 2007 a Dezembro de 2008. A ideia foi verificar se o fato de os dois rios se localizarem na mesma bacia hidrográfica é suficiente para que se considere a aplicação de um único plano de manejo para ambos. Os dados mostraram que os rios apresentam significativa interferência antrópica. Os resultados sugeriram que tais rios devem ser submetidos a planos individuais de gerenciamento, uma vez que houve ocorrências exclusivas (variáveis que foram detectadas em apenas um dos rios). Além disso, houve diferença estatisticamente significativa entre os períodos seco e chuvoso para onze variáveis ( $\mathrm{p}^{*}<0,05$, ANOVA), o que reforça a especial importância da componente temporal do programa de monitoramento. Espera-se que esse estudo ofereça subsídios para programas de recuperação ambiental do rio Tietê, para o qual é recomendado foco na repressão de lançamentos clandestinos de águas residuárias, e do rio Pinheiros, para o qual se sugere especial atenção à poluição derivada do aporte de pesticidas ao corpo de água.

Palavras-chave: monitoramento ambiental, região metropolitana de São Paulo (RMSP), rios urbanos, poluição da água, qualidade da água, gestão de bacias hidrográficas.

\section{REFERENCES}

Abraham W, Macedo AJ, Gomes lH and Tavares FCA. 2007. Occurrence and resistance of pathogenic bacteria along the Tiete River downstream of São Paulo in Brazil. Clean - Soil Air Water 35: 339-347.

Andrade CFS, CAmpos J, CABrini I, Marques-Filho CAM AND HIBI S. 2007. Susceptibilidade de populações de Culex quinquefasciatus Say (Diptera: Culicidae) sujeitas ao controle com Bacillus sphaericus Neide no rio Pinheiros, São Paulo. BioAssay 2: 1-4.

APHA - American Public Health Association. 2005. Standard Methods for the examination of water and wastewater. $25^{\text {th }}$ ed., Washington: APHA.

ATSDR - AGENCY FOR TOXIC SUbSTANCES AND DisEASE REgISTRY. 1999. Toxic facts and information. Available from: <http://www.atsdr.cdc.gov>. Access: 03/06/2009.

Bedore PD, David MB And Stucki JW. 2008. Mechanisms of phosphorus control in urban streams receiving sewage effluent. Water Air Soil Poll 191: 217-229. 
Blanchoud H, Moreau-Guigon E, Farrugia F, CheVReuil M AND Mouchel JM. 2007. Contribution by urban and agricultural pesticide uses to water contamination at the scale of the Marne Watershed. Sci Total Environ 375: 168-179.

Bracco JE, Dalbon M, Marinotti O AND Barata JM. 1997. Resistance to organophosphorous and carbamates insecticides in a population of Culex quinquefasciatus. Rev Saúde Pública 31: 182-183.

Bradley PM, Chapelle FH AND LOVley DR. 1998. Humic acids as electron for anaerobic microbial oxidation of Vinyl Chloride and Dichloroethene. Appl Environ Microb 64: 3102-3105.

BRAGA BPF. 2000. The management of urban water conflicts in the Metropolitan Region of São Paulo. Water Int 25: 208-213.

DAVYDOVA S. 2005. Heavy metals as toxicants in big cities. Microchem J 79: 133-136.

Duhamel M, Wehr SD, Yu L, Rizvi H, Seepersad D, Dworatzek S, COX EE AND Edwards EA. 2002. Comparison of anaerobic dechlorinating enrichment cultures maintained on tetrachloroethene, trichloroethene, cis-dichloroethene and vinyl chloride. Water Res 36: 4193-4202.

Franco RMB, Rocha-Eberhardt R AND CANTUSIONETO R. 2001. Occurrence of Cryptosporidium oocysts and Giardia cysts in raw water from the Atibaia River, Campinas, Brazil. S. Paulo. Rev Inst Med Trop 43: 109111.

Fuchs S, HARitopoulou T, SCHAFER M AND Wilhelmi M. 1997. Heavy metals in freshwater ecosystems by urban rainwater runoff - monitoring of suspended solids, river sediments and biofilms. Water Sci Technol 36: 277-282.

Galbraith LM AND BURNS CW. 2007. Linking landuse, water body type and water quality in southern New Zealand. Landscape Ecol 22: 231-241.

Göbel P, Dierkes C ANd Coldewey WG. 2007. Storm water runoff concentration matrix for urban areas. J Contam Hydrol 91: 26-42.

GRAY L. 2004. Changes in water quality and macroinvertebrate communities resulting from urban stormflows in the Provo River, Utah, USA. Hydrobiologia 518: 33-46.

Green T, LEe R, FARRAR D AND HILL J. 2003. Assessing the health risks following environmental exposure to hexachlorobutadiene. Toxicol Letters 138: 63-73.

HACHICH EA. 2002. Avaliação da presença dos protozoários Giardia sp. e Cryptosporidium sp. em águas superficiais destinadas a captação e tratamento para consumo humano no Estado de São Paulo. Universidade de São Paulo USP, São Paulo. [PhD thesis in Public Health].

Heller L, Bastos RKX, Vieira MBCM, Bevilacqua PD, Brito LlA, Mota SMM, Oliveira AA, MACHAdo PM, SALVAdor DP AND CARdoso AB. 2004. Oocistos de Cryptosporidium e cistos de Giardia: circulação no ambiente e riscos à saúde humana. Epidemiol Serv Saúde 13: 79-92.

HWANG HM AND FOSTER GD. 2006. Characterization of polycyclic aromatic hydrocarbons in urban stormwater runoff flowing into the tidal Anacostia River, Washington, DC, USA. Environ Pollut 140: 416-426.

Juang DF, Yuan CS, Hsueh SC and Chiou LJ. 2009. Distribution of volatile organic compounds around a polluted river. Int J Environ Sci Te 6: 91-104.

Kolpin DW, Thurman EM And Linhart SM. 1998. The environmental occurrence of herbicides: the importance of degradates in ground water. Arch Environ Contam Toxicol 35: 385-390.

KREIN AS AND SCHORER M. 2000. Road runoff pollution by polycyclic aromatic hydrocarbons and its contribution to river sediments. Water Res 34: 4110-4115.

Manoli E, Samara C, Konstantinou I and AlbaNIS T. 2000. Polycyclic aromatic hydrocarbons in the bulk precipitation and surface waters of Northern Greece. Chemosphere 41: 1845-1855.

MCCUlloch A. 2003. Chloroform in the environment: occurrence, sources, sinks and effects. Chemosphere 50: 1291-1308.

Miserendino ML, BRAND C AND PRinZio CYD. 2008 Assessing urban impacts on water quality, benthic communities and fish in streams of the Andes Mountains, Patagonia (Argentina). Water Air Soil Poll 194: 91-110.

Morais SA, Marrelli MT and Natal D. 2006. Aspectos da distribuição de Culex (Culex) quinquefasciatus Say (Diptera, Culicidae) na região do rio Pinheiros, na cidade de São Paulo, Estado de São Paulo, Brasil. Rev Bras Entomol 50: 413-418.

Morais SA, Marreli MT AND NATAL D. 2007. Observações sobre aplicações de controle em ecótopo com alta infestação de Culex quinquefasciatus Say (Diptera, Culicidae). Rev Bras Entomol 51: 246-251.

Muneer M, Singh HK and Bahnemann D. 2002. Semicondutor-mediated photocatalysed degradation of two selected priority organic pollutants, bendizine and 1,2diphenylhydrazine, in aqueous suspension. Chemosphere 49: 193-203. 
PANNA - Pesticide ACtion Network North AmerICA. 2009. Pesticide Database - Chemicals. Identification, toxicity, use, water pollution potential, ecological toxicity and regulatory information. Available from: $<$ http://www.pesticideinfo.org >. Access: 03/06/2009.

Silva IS, Abate G, Lichtig J And Masini JC. 2002. Heavy metal distribution in recent sediments of the TietêPinheiros river system in São Paulo state, Brazil. Appl Geochem 17: 105-116.

Silva-FilHa MHNL ET AL. 2008. Culex quinquefasciatus field populations subjected to treatment with Bacillus sphaericus did not display high resistance levels. Biol Control 44: 227-234.

Staples B, Howse MLP, Mason H ANd Bell GM. 2003. Land contamination and urinary abnormalities: cause for concern? Occup Environ Med 60: 463-467.

Tchounwou PB, Abdelghani AA, Pramar YV And HEYER LR. 1998. Health risk assessment of hexachlorobenzene and hexachlorobutadiene residues in fish collected from a hazardous waste contaminated wetland in Louisiana, USA. Am Soc Test Mater 1333: 368-382.
TONG STY AND CHEN W. 2002. Modeling the relationship between land use and surface water quality. J Environ Manage 66: 377-393.

TOXMAP Chemical Page. 2009. Toxicology database. Available from: <http://toxmap.nlm.nih.gov>. Access: 03/06/2009.

TUCCI CEM. 2004. Gerenciamento integrado das inundações urbanas no Brasil. Revista de Gestão de Água da América Latina 1: 59-73.

TURNER RE AND RABALAIS NN. 2003. Linking landscape and water quality in the Mississippi River Basin for 200 years. Bioscience 53: 563-572.

VARIS O, Biswas AK, TORTAJAdA C AND LUNDQVist J. 2006. Megacities and water management. Water Resources Development 22: 377-394. 